\title{
CRESCIMENTO DE LEGUMINOSAS ARBÓREAS EM RESPOSTAS A INOCULAÇÃO COM FUNGOS MICORRÍZICOS ARBUSCULARES
}

\section{THE GROWTH OF ARBOREAL LEGUMINOUS IN ANSWER TO INOCULATION WITH ARBUSCULAR MYCORRHIZAL FUNGI}

\author{
Marcos Vinicius Winckler Caldeira ${ }^{1}$ Eliane Maria Ribeiro da Silva ${ }^{2}$ Avílio A. Franco ${ }^{2}$ \\ Magda Lea Bolzan Zanon ${ }^{3}$
}

\begin{abstract}
RESUMO
O presente estudo teve como objetivo avaliar o desenvolvimento de mudas inoculadas ou não com fungos micorrízicos. O delineamento experimental utilizado foi inteiramente casualizado com 4 tratamentos e 25 repetições. Os tratamentos foram: Glomus clarum (Nicolson \& Schenk), Gigaspora margarita (Becker Hall), Fungos Nativos e Testemunha (sem inoculação). Não houve diferença estatística para altura, diâmetro e fitomassa aérea e subterrânea de raízes grossas em mudas de Copaifera martii. As maiores percentagens de comprimento de raízes finas colonizadas e taxa de sobrevivência foram obtidas com mudas inoculadas com G. margarita e G. clarum. No que se refere as mudas de Dimorphandra macrostachya não houve diferença estatística para os parâmetros estudados (altura, diâmetro, fitomassa aérea e subterrânea de raízes grossas e finas. A inoculação com $G$. margarita favoreceu a percentagem de colonização micorrrízica de raízes finas e a taxa de sobrevivência em mudas de Dimorphandra macrostachya.
\end{abstract}

Palavras-chave: Fungos Micorrízicos, Árvores Leguminosas, Crescimento.

\begin{abstract}
The present study aimed at evaluating the development of inoculated or noninoculated seedlings with mycorrhizal fungi. The experimental design used was completely randomized, with 4 treatments and 25 replacements. The treatments were: Glomus clarum (Nicolson \& Schenk),

1. Eng. Florestal, Pós-Graduando em Engenharia Florestal, CCR/UFSM. Universidade Federal de Santa Maria. CEP: 97119-900. Santa Maria. RS.

2. Doutores e Pesquisadores do Centro Nacional de Pesquisa Agrobiológica - CNPAB/EMBRAPA. Antiga Rodovia Rio/SP, CEP: 23850-970. Seropédica/Itaguaí. RJ.

3. Eng. Florestal, Mestre em Manejo Florestal, CCR/UFSM. Universidade Federal de Santa Maria. CEP: 97119-900. Santa Maria. RS.
\end{abstract}


Gigaspora margarita (Becker \& Hall), Native Fungi and Control (without inoculation). There was no statistical difference for height, diameter, aerial and subterranean phytomasses of thick roots in Copaifera martii seedlings. The highest length percentage of colonized thin roots and the survival rate were obtained in inoculated seedlings with G. margarita and G. clarum. Concerning the seedlings of Dimorphandra macrostachya, there was no statistical difference for height, diameter, aerial and subterranean phytomasses of thick or thin roots. The inoculation with G. margarita favored the percentage of mycorrhizal colonization of thin roots and survival rate in Dimorphandra macrostachya seedlings.

Key words: Mycorrhizal fungi, Leguminous trees, growth.

\section{INTRODUÇÃO}

A matéria orgânica do solo é a principal fonte de nutrientes minerais para as plantas. Assim, a perda da fertilidade natural dos solos tropicais úmidos tem como principal causa o desaparecimento da matéria orgânica do mesmo.

Desta forma, nestas regiões, toda exploração agrícola, deve ter como maior preocupação um manejo conservacionista da matéria orgânica do solo. A sua perda, causa sérios problemas na estrutura do solo, disponibilidade de água, atividade biológica do mesmo, prejudica o suprimento de enxofre, fósforo e principalmente de nitrogênio às plantas e como conseqüência, a produtividade do sistema. Este processo é mais intenso em áreas de declividade acentuada onde além da decomposição natural da matéria orgânica, há perdas, pela remoção da parte superficial do solo por erosão. O caso extremo, ocorre com a movimentação de terras, como nas construções de barragens estradas, áreas de mineração, etc, onde todo horizonte superfícial é removido.

A revegetação destas áreas geralmente é feita com alto investimento, muitas vezes às custas da transferência da camada fértil de outras áreas. Uma opção com menos investimento na revegetação destas áreas é utilizar espécies leguminosas noduladas e micorrizadas associadas à adubação com gesso, fosfato de rocha e quando disponível, composto orgânico (FRANCO et al., 1991).

O enxofre e o fósforo podem ser supridos às plantas por fontes pouco solúveis e de disponibilidade a longo prazo (ex: sulfato de cálcio e fosfato natural); enquanto que, o nitrogênio pode ser fornecido em forma contínua, através do uso de bactérias fixadoras de nitrogênio atmosférico associadas, a algumas espécies vegetais. Entre as espécies pioneiras, as leguminosas despertam grande interesse, já que em sua maioria, são lenhosas e perenes e formam simbiose com rizóbio, que fixa nitrogênio do ar. Essas espécies, quando associadas a fungos micorrízicos, aproveitam melhor o fósforo e outros nutrientes do solo. As leguminosas arbóreas contribuem para a recuperação do solo pela deposição de folhedo com baixa relação $\mathrm{C} / \mathrm{N}$ e pela ação das raízes.

A deposição das folhas e o crescimento das raízes estabilizam o solo, aumentam a atividade biológica do mesmo e criam condições propícias para o estabelecimento de outras espécies mais exigentes. As leguminosas são ainda, importantes produtoras de lenha, carvão, madeira, postes, ferragem, celulose, tanino e outros produtos.

Ciência Florestal, v.7, n.1, 1997 
A recuperação de solos degradados deve ser baseada numa tecnologia que, promova não apenas a utilização de espécies de rápido crescimento, mas também que sejam capazes de melhorar o solo através da matéria orgânica. Esta melhoria se faz tanto pela deposição de material vegetal como reciclagem de nutrientes (MONTAGNINI \& SANCHO, 1990).

Grande parte das leguminosas apresentam uma elevada produção de biomossa com um significativo aporte de folhas ao solo. Estimativas na Região Nordeste indicam que 5,8 t/ha/ano de material de sabiá (Mimosa caesalpiniifolia), podem retornar ao solo (SUASSUNA, 1982). Em condições climáticas similares, a Leucaena leucocephala, contribui com um aporte na ordem de 10t/ha/ano (SANDHU et al., 1990 ). Em sistema de sombreamento para café na América Central, a Erythrina sp. contribuim com um aporte de até 13,5 t/ha/ano de material vegetal ( GLOVER \& BEER, 1986 ). Desta forma as espécies fixadoras de nitrogênio podem apresentar uma significativa melhoria na fertilidade do solo.

A grande maioria das espécies florestais, quando na sua fase de muda, necessita de maior disponibilidade de fósforo pois a demanda deste nutriente é mais intensa (NOVAIS et al., 1990). As leguminosas dependendo da simbiose como fonte de nitrogênio, requerem alto teor de fósforo na solo para suprir as necessidades adicionais dos nódulos. Além disto a presença de nódulos pode diminuir o crescimento de raízes quando comparadas com plantas que recebem o $\mathrm{N}$ mineral (CASSMANN et al., 1981).

O presente estudo teve por objetivo estudar a influência dos fungos micorrízicos arbusculares no desenvolvimento de leguminosas arbórea.

\section{REVISÃO BIBLIOGRÁFICA}

A micorriza forma uma associação íntima e benéfica com as plantas, que resulta no aumento da absorção de água e nutrientes pelos vegetais, principalmente o fósforo, devido sua baixa mobilidade no solo. As associações micorrízicas são de três tipos: endomicorrizas, ectomicorrizas e ectendomicorrizas. O grupo das endomicorrizas arbusculares (MA) apresentam colonização nos espaços inter e intracelulares das células da epiderme e córtex das raízes, formando arbúsculos. Além disso, apresenta grande quantidade de micélio externo que pode atingir sítios fora da zona de depleção de nutrientes, aumentando assim absorção e o aproveitamento de água e nutrientes da solução do solo.

Os fungos micorrízicos arbusculares (FMA) são biotróficos abrigatórios, ou seja, apenas crescem e esporulam na presença de raízes vivas, o que faz com que sua utilização em larga escala na agricultura seja limitada pela falta de inoculante aceito comercialmente e de um padrão oficial para seu controle de qualidade.

Embora os estádios de sucessão estejam bem definidos nas florestas tropicais, são raros os estudos sobre os mecanismos da sucessão, principalmente daqueles relacionados aos aspectos nutricionais das espécies. SIQUEIRA et al.(1994) têm resultados preliminares sobre a avaliação da resposta de espécies arbóreas, a maioria nativas do sudeste brasileiro, pertencente a diferentes grupos sucessionais, a adição de fósforo (P) e inoculação com os FMAs ( Glomus etunicatum e 
Gigaspora margarita). Foram estudadas trinta e uma espécies, sendo seis pioneiras, nove secundárias inicias, nove secundárias tardias e sete climax. Todas as pioneiras mostraram-se micotróficas com colonização em torno de $60 \%$, enquanto seis das tardias e climax não apresentam colonização. As respostas das pioneiras a inoculação com FMAs e o P, foram em média 4250\% e $3739 \%$ respectivamente, enquanto para as demais estes valores foram pouco mais de $1000 \%$ para secundárias e em torno de $100 \%$ para as climax. Tendências semelhante foram verificadas para respostas a $\mathrm{P}$ mais FMAs.

Os efeitos dos fungos arbusculares sobre o crescimento da planta hospedeira, podem ser observados mediante experimento realizados em casa de vegetação, onde há possibilidade de obter plantas não-micorrizadas que serão comparadas com aquelas micorrizadas. Quando estes experimentos são conduzidos em recipientes, pode-se observar que diferentes espécies de FMA, testados com o mesmo hospedeiro sob as mesmas condições ambientais, podem ter diferentes habilidades na formação do crescimento desse hospedeiro (SANDERS, 1977).

\section{MATERIAL E MÉTODOS}

O presente trabalho foi conduzido em casa de vegetação localizada, no Centro Nacional de Pesquisa de Agrobiologia (EMBRAPA/CNPAB) Itaguai, RJ com $22^{\circ} 46^{\prime}$ de latitude Sul e $43^{\circ}$ 41 'de longitude Oeste com altitude de 33 metros. De acordo com KOEPPEN o clima predominante na região é AW.

As sementes das espécies de Copaifera martii (copaíba) e Dimorphandra macrostachya (fava-pomba) foram provenientes da região de Porto Trombetas-PA. As sementes passaram por um processo de quebra de dormência em ácido sulfúrico (95-97 \% PA) permanecendo no mesmo por 35 minutos para Copaifera martii e 60 minutos para Dimorphandra macrostachya, após as mesmas foram desinfestadas com Peróxido de Hidrogênio (30 \% PA) por dois minutos e em seguida lavadas com água estéril.

Após a quebra de dormência as sementes foram colocadas em placas de Petri esterilizadas e levadas ao germinador, a uma temperatura de $34,4^{\circ} \mathrm{C}$ permanecendo de dois a três dias.

O substrato utilizado foi uma mistura de composto orgânico : argila : areia: fosfato de rocha natural na proporção de 6:2:1:1 respectivamente. Para a esterilização do substrato utilizou-se 0,60 $\mathrm{ml}$ de brometo de metila/Kg de solo permanecendo hermeticamente fechado durante 96 horas.

O plantio das sementes pré-germinadas foi feito em bandejas de isopor com 72 células e em cada bandeja foram utilizadas 25 células, sendo que em cada célula foram colacadas 02 sementes e quando as plântulas obtiveram dois pares de folhas definitivas foi realizado desbastes deixando uma plântula por célula.

Juntamente no momento do plantio das sementes pré-germinadas foi realizada a inoculação com fungos micorrízicos arbusculares. Em cada célula foram colocados 30 esporos/planta.

O delineamento experimental utilizado foi inteiramente casualizado com 4 tratamentos e 25 repetições. Os tratamentos foram: Glomus clarum (Nicolson \& Schenk) Gigaspora margarita 
(Becker \& Hall), Fungos Nativos e Testemunha (sem inoculação). O solo do tratamento testemunha foi desinfestado com brometo de metila $(0,60 \mathrm{ml}$ de brometo de metila/ $\mathrm{Kg}$ de solo) Os fungos nativos foram procedentes da rizosfera de plantas mata virgem da região de Porto Trombetas-PA.

Após 168 dias foram avaliados os parâmetros: altura, diâmetro à altura do colo, peso da parte aérea e raiz seca e percentagem do comprimento de raízes colonizadas.

Do volume de solo coletado em cada célula foram separadas raízes finas $(<1,0 \mathrm{~mm}$ de diâmetro) para avaliação da colonização micorrízica.

Para verificação da colonização micorrízica, o sistema radicular foi lavado e colocado em papel absorvente para ser retirado o excesso de umidade. Foi retirado $0,5 \mathrm{~g}$ de raízes finas, onde as mesmas foram lavadas com água destilada e conservadas em etanol 50\%. O clareamento e coloração das raízes foi feito de acordo com a metodologia propostas por KOSKE \& GEMMA (1989).

A percentagem do comprimento de raízes finas colonizadas foi avaliado pelo método da placa quadriculada (GIOVANNETTI \& MOSSE, 1980).

\section{RESULTADOS E DISCUSSÃO}

\section{a) Copaifera martii}

Não houve diferença significativa entre os tratamentos com relação a altura, diâmetro, fitomassa aérea e fitomassa subterrânea de raízes grossas (Tabela 1).

TABELA 1: Altura (h), diâmetro à altura do colo (d), peso da fitomassa aérea (FA), fitomassa subterrânea de raízes grossas - FSRG ( $>$ que 1,0mm de diâmetro) e fitomassa subterrânea de raízes finas - FSRF ( $<$ que 1,0mm de diâmetro) em mudas de Copaifera martii, inoculadas ou não com FMA, aos 168 dias após a germinação.

\begin{tabular}{l|c|c|c|c|c}
\hline Tratamentos & $\mathrm{h}(\mathrm{cm})$ & $\mathrm{d}(\mathrm{cm})$ & $\begin{array}{c}\text { FA } \\
\text { g. planta }\end{array}$ & $\begin{array}{c}\text { FSRG } \\
\text { g. planta }\end{array}$ & $\begin{array}{c}\text { FSRF } \\
\text { g. planta }\end{array}$ \\
\hline G. clarum & $10,21 \mathrm{a}^{-1}$ & $0,23 \mathrm{a}^{1}$ & $0,36 \mathrm{a}^{1}$ & $0,26 \mathrm{a}^{1}$ & $0,06 \mathrm{a}^{1}$ \\
G. margarita & $11,57 \mathrm{a}$ & $0,23 \mathrm{a}$ & $0,40 \mathrm{a}$ & $0,28 \mathrm{a}$ & $0,02 \mathrm{~b}$ \\
Fungos Nativos & $9,55 \mathrm{a}$ & $0,20 \mathrm{a}$ & $0,28 \mathrm{a}$ & $0,18 \mathrm{a}$ & $0,03 \mathrm{ab}$ \\
Testemunha & $10,36 \mathrm{a}$ & $0,21 \mathrm{a}$ & $0,32 \mathrm{a}$ & $0,25 \mathrm{a}$ & $0,05 \mathrm{ab}$ \\
\hline CV $(\%)$ & 26,07 & 13,79 & 40,73 & 36,29 & 38,64 \\
\hline
\end{tabular}

${ }^{1}$ Tratamentos com médias não ligadas por mesma letra diferem pelo teste Tukey em nível 5\% de probabilidade de erro.

Observa-se na Tabela 1 que mudas inoculadas com G. clarum apresentaram maior acúmulo 
de fitomassa subterrânea de raízes finas.

SAIF (1987) verificou que em forrageiras tropicais, a inoculação com FMVA favoreceu mais o desenvolvimento das partes aéreas do que de raízes levando a um significativo decréscimo na relação raiz/parte aérea em quartoze entre vinte e uma espécies, sendo que algumas, a inoculação não teve efeito ou aumentou esta relação.

$\mathrm{Na}$ Tabela 2 pode-se observar que as maiores percentagens de colonização micorrízica foram obtidas com G. margarita e G. clarum, diferenciando significativamente de fungos nativos e testemunha. As maiores taxas de sobrevivência foram observadas nas mudas inoculadas com $G$. margarita e G. clarum

TABELA 2: Percentagem do comprimento de raízes finas (< que 1,0mm de diâmetro) colonizadas PCRFC com FMA e taxa de sobrevivência - TS em mudas de Copaifera martii, aos 168 dias após a germinação.

\begin{tabular}{l|c|c}
\hline Tratamentos & PCRFC (\%) & TS (\%) \\
\hline G. clarum & $32,20 \mathrm{a}^{1}$ & 44 \\
G. margarita & $33,40 \mathrm{a}$ & 48 \\
Fungos nativos & $17,38 \mathrm{~b}$ & 28 \\
Testemunha & $0,00 \mathrm{c}$ & 23 \\
\hline
\end{tabular}

${ }^{1}$ Tratamentos com médias não ligadas por mesma letra diferem pelo teste Tukey em nível 5\% de probabilidade de erro.

De acordo com DAFT \& NICOLSON (1966) a obtenção de diferenças consistentemente altas e significativas entre plantas micorrizadas e não micorrizadas, leva em consideração dois fatores: a disponibilidade de nutrientes no solo e o nível de colonização da raiz. Segundo os mesmos autores, os efeitos da associação micorrízica sobre o crescimento da planta depende, de alguma forma, do balaço entre esses dois fatores.

PARON et al. (1994) estudou através de dois experimentos a resposra de Senna macranthera, Rich e Cipaifera languisdorfii Desf. ao fósforo e nitrogênio e Glomus etunicatum. Os autores verificaram que no segundo experimento o fedegoso (Senna macranthera) apresentou diferença para inoculação e adição de $\mathrm{P}$ não apresentando diferença para $\mathrm{N}$ e fumigação. $\mathrm{O}$ óleo-decopaíba (Copaifera languisdorfii) apresentou respostas variadas aos diversos tratamentos, mostrando efeito negativo da inoculação nos dois experimento.

PEREIRA et al. (1994b) avaliaram a resposta da cassia-verrugosa (Senna multijuga), fedegoso (Senna macranthera), óleo-de-copaíba (Copaiba languisdorfii), tamboril (Enterolobium contortisiliqum) e angico-vermelho (Anadenanthera peregrina) á adubação nitrogenada e inoculação com Glomus etunicatum. Os mesmos autores verificaram que para todos os parâmetros (altura de plantas, peso de matéria seca, diâmetro de caule e colonização), efeitos significativos para ambos fatores, para todas as espécies, com exceção do óleo-de-copaíba que não respondeu positivamente à nenhum dos fatores.

Ciência Florestal, v.7, n.1, 1997 


\section{b) Dimorphandra macrostachya}

Análise estatística dos dados de altura, diâmetro, fitomassa aérea e subterrânea, de raízes finas e grossas não mostraram diferença significativa entre os tratamentos (Tabela 3 ).

Com o objetivo de avaliar o melhor momento de inoculaçao com FMVA (G. clarum e $G$. margarita) e seu efeito no desenvolvimento inicial de acerola (Malpighia emarginata D.C.) PEREIRA et al. (1994) verificaram que aos 56 dias após a semeadura, não houve respostas significativas na altura, diâmetro à altura do colo das plantulas e peso da parte aérea seca. Três meses após a repicagem das mudas, as plantas inoculadas nesta etapa apresentaram em média, maior altura e mais matéria seca de raiz do que as não inoculadas. $\mathrm{O}$ fungo que produziu maior número de esporos foi G. margarita. A resposta da acerola à colonização micorrízica foi pequena na fase das mudas.

TABELA 3: Altura (h), diâmetro à altura do colo (d), peso da fitomassa aérea (FA), fitomassa subterrânea de raízes grossas - FSRG ( $>$ que 1,0mm de diâmetro) e fitomassa subterrânea de raízes finas - FSRF ( $<$ que 1,0mm de diâmetro) em mudas de Dimorphandra macrostachya, inoculadas ou não com FMA, aos 168 dias após a germinação.

\begin{tabular}{l|c|c|c|c|c}
\hline Tratamentos & $\mathrm{h}(\mathrm{cm})$ & $\mathrm{d}(\mathrm{cm})$ & $\begin{array}{c}\text { FA } \\
\text { g. planta }\end{array}$ & $\begin{array}{c}\text { FSRG } \\
\text { g. planta }\end{array}$ & $\begin{array}{c}\text { FSRF } \\
\text { g. } \text { planta }^{-1}\end{array}$ \\
\hline G. clarum & $8,81 \mathrm{a}^{1}$ & $0,27 \mathrm{a}^{1}$ & $0,36 \mathrm{a}^{1}$ & $0,14 \mathrm{a}^{1}$ & $0,12 \mathrm{a}^{1}$ \\
G. margarita & $9,28 \mathrm{a}$ & $0,27 \mathrm{a}$ & $0,32 \mathrm{a}$ & $0,13 \mathrm{a}$ & $0,09 \mathrm{a}$ \\
Fungos Nativos & $9,78 \mathrm{a}$ & $0,27 \mathrm{a}$ & $0,40 \mathrm{a}$ & $0,13 \mathrm{a}$ & $0,12 \mathrm{a}$ \\
Testemunha & $8,00 \mathrm{a}$ & $0,25 \mathrm{a}$ & $0,28 \mathrm{a}$ & $0,08 \mathrm{a}$ & $0,09 \mathrm{a}$ \\
\hline CV $(\%)$ & 17,87 & 17,26 & 40,73 & 52,29 & 48,57 \\
\hline
\end{tabular}

${ }^{1}$ Tratamentos com médias não ligadas por mesma letra diferem pelo teste Tukey em nível 5\% de probabilidade de erro.

ROCHA et al. (1994) estudaram os efeitos de doses de P e da inoculação com FMVA sobre os teores de $\mathrm{P}, \mathrm{Ca}, \mathrm{Mg}, \mathrm{S}, \mathrm{Cu}$ e $\mathrm{Mn}$ no crescimento de Citrus reshni Hort ex Tan, os autores verificaram que a inoculação com Acaulospora morrowae, G. clarum e G. etunicatum promoveram maior produção de matéria seca da parte aérea e da raiz e a diluição dos teores de $\mathrm{Ca}, \mathrm{Mg}, \mathrm{Cu}$ e $\mathrm{Mn}$.

Em relação ao comprimento de raízes finas colonizadas com FMA as maiores percentagens de colonização micorrízica foram obtidas com fungos nativos e G. margarita, diferenciando significativamente de $G$. clarum e testemunha. A maior taxa de sobrevivência foi observado nas mudas inoculadas com G. margarita (Tabela 4). 
TABELA 4: Porcentagem do comprimento de raízes finas (< que 1,0mm de diâmetro) colonizadas PCRFC com FMA e taxa de sobrevivência - TS em mudas de Dimorphandra macrostachya, aos 168 dias após a germinação.

\begin{tabular}{c|c|c}
\hline Tratamentos & PCRFC (\%) & TS (\%) \\
\hline G. clarum & $14,27 \mathrm{~b}^{1}$ & 48 \\
G. margarita & $19,37 \mathrm{a}$ & 76 \\
Fungos Nativos & $20,17 \mathrm{a}$ & 44 \\
Testemunha & $0,00 \mathrm{c}$ & 44 \\
\hline
\end{tabular}

${ }^{1}$ Tratamentos com médias não ligadas por mesma letra diferem pelo teste Tukey em nível 5\% de probabilidade de erro.

As respostas positivas de crescimento em relação à colonização micorrízica são normalmente interpretados como efeito da melhora na nutrição mineral das plantas, pelo aumento na absorção de nutrientes, principalmente o $\mathrm{P}$, pelas raízes micorrizadas, resultantes da produção de substâncias reguladoras de crescimento pelo fungo (SMITH, 1980; HAYMAN, 1983; MENGE, 1983 ) melhora na absorçãode água, fixação de nitrogênio e resistência a doenças da raiz (DAFT \& NICOLSON, 1966; GIANINAZZI-PEARSON \& GIANINAZZI, 1983).

\section{CONCLUSÕES}

Com base nos resultados apresentados pode-se concluir:

As maiores percentagens de colonização micorrízica e taxa de sobrevivência em mudas de Copaifera martii foram obtidas, respectivamente, com G. margarita e G. clarum.

A inoculação com G. margarita, respectivamente, favoreceu a percentagem de colonização micorrízica de raízes finas e a percentagem de sobrevivência em muda de Copaifera martii e Dimorphandra macrostachya.

Mudas de Copaifera martii e Dimorphandra macrostachya inoculadas com G. clarum não tiveram incrementos nos parâmetros avaliados (altura, diâmetro, fitomassa aérea e fitomassa subterrânea de raízes finas e grossas.

\section{REFERÊNCIAS BIBLIOGRÁFICAS}

CASSMANN, K. G.; WHITNEY, A. S.; FOX, P.L. Phosphorus requirements of cowpea and soybeans as affected by made of N nutrition. Agron. J. v.73 p.17-22, 1981.

DAFT, M.J., NICOLSON, T.H. Effect of Endogone mycorrhiza on plant growth. The New Phytologist, Cambridge, v.65, n.3, p.343-350, 1966. 
FRANCO, A.A.; CAMPOS NETO, D.; CUNHA, C. de O.; CAMPELLO, E. F. C.; MONTEIRO, E. M. da S.; SANTOS, C.J. F.; FONTES, A. M.; FARIA, S. M. de. Revegetação de solos degradados. In: WORKSHOP SOBRE RECUPERAÇÃO DE ÁREAS DEGRADADAS,1 1990, Itaguaí. Anais... Itaguaí: UFRRJ/Departamento de Ciências Ambientais, 1991. p.133-157.

GIANINAZZI-PEARSON, V.; GIANINAZZI, S. The physiology of vesicular-arbuscular mycorrhizal roots, Plant and Soil, The Rague, v.71, p.197-209, 1983.

GIOVANNETTI, M.; MOSSE, B. An evaluation of techniques for measurig VA mycorrhizal infection in roots. New Phytol. v.84, p.489-500, 1980.

GLOVER, N.; BEER, J. Nutrient cycling in two traditional central american agroforesty. Agrof. Syst. v.4, p.77-87, 1986.

HAYMAN, D.S. The physiology of vesicular-arbuscular endomycorrhizal symbiosis. Canadian Journal of Botany, Ottawa, v.61, p.944-963, 1983.

KOSKE, R.E.; GEMMA,J.N. A modified procedure pr staining roots to detect VA mycorrhizas. Micol. Res., v.92 p. 488-505, 1989.

MENGE, J.A. Utilization of vesicular-arbuscular mycorrhizal fungi in agriculture. Canadian Journal of Botany, Ottawa, v.61, p.1015-1024, 1983.

MONTAGNINI, E. M.; SANCHO, F. Impacts of native trees on tropical soils: a study in the Atlantic lawlands of Costa Rica. Central America. Ambio. v.19, p.386-390, 1990.

NOVAIS, R.F.; BARROS, N.F.; NEVES, J.C.L. Nutrição mineral do eucalipto. In: BARROS, N.F.; NOVAES, R. F. (eds). Relação solo-eucalipto. Viçosa. Folha de Viçosa. p.25-98. 1990.

PARON, M.E.; SIQUEIRA, J.O.; VALE, F.R. \& CURI, N. Resposta de duas leguminosas arbóreas ao fósforo, nitrogênio e fungo endomicorrízico. In: REUNIÃO BRASILEIRA SOBRE MICORRIZAS, 1994. Resumos... Florianópolis: REBRAM, 1994 p.51

PEREIRA, E.G.; SIQUEIRA, J.O., VALE, F.R.; CURI, N. ; MOREIRA, F.M.S. respostas de Leguminosas arbóreas nativas da região dos Campos das vertentes (MG) à adubação nitrogenada e fungos endomicarrízicos. In: SIMPÓSIO BRASILEIRO SOBRE MICROBIOLOGIA DO SOLO, 1994. Resumos... Londrina: SBMS,1994b 162p. p109.

PEREIRA, M.G.; SILVA, E.M.R. da.; SUDO, A.; LEAL, M.A. de. A. Micorrizas vesículoarbusculares em acerola (Malpighia emarginata D.C.) efeito da inoculação na fase de produção de mudas. In: REUNIÃO BRASILEIRA SOBRE MICORRIZAS, 1994. Resumos... Florianópolis: REBRAM, 1994 p.57.

ROCHA, M.R.da.; CORRÊA, G.C.; OLIVEIRA, E. de. Efeito de micorrizas e da adubação fosfatada sobre a tangerineira "cleopatra" (Citrus reshni Hort ex Tan.). In: REUNIÃO BRASILEIRA SOBRE MICORRIZAS, 1994. Resumos... Florianópolis: REBRAM, 1994. p.62.

SAIF, S.R; Growth responses of tropical forage plant species to vesicular-arlbuscular mycorrhizae. Plant and soil , Dordrecht, v.97, p.25-35, 1987.

SANDHU, J.; SINHA, M.; AMBASHT, R. S. Nitrogen release from decomposing litter of Ciência Florestal, v.7, n.1, 1997 
Leucaena leucocephala in the dry tropics. Soil.Biol. Biochem. v.22 n.6 p. 859-863. 1990.

SANDERS, F.E.; TINKER, P.B.; BLACK, R.L.B.; PALMERLEY, S.M.The development of endomycorrhizal root systems. 1 .Spread on infection and growth-fromoting effects with four species of vesicular-arbuscular endophyte.The New Phytoligist, Cambridge, v.78, p.257-268, 1977.

SIQUEIRA,J.O.; CARNEIRO, M.A.C.; DAVIDE, A.C.; CURI, N.; VALE F.R. Resposta à fósforo e fungos micorrízicos arbusculares (FMAs) por espécies florestais de diferentes grupos sucessionais. In: REUNIÃO BRASILEIRA SOBRE MICORRIZAS, 1994. Resumos... Florianópolis: REBRAM, 1994 p.20.

SMITH, S.E. Micorrizas of autotrophic higler plants. Biological Reviews, Cambridge, v.55, p.475$510,1980$.

SUASSUNA,J. Efeitos da Associação do sabiá (Mimosa Caesalpiniifolia Benth.) no comportamento do Jacarandá (Balbergia nigra, Fr Allen) e da peroba-branca (Tabebuia stenocalyx Sprague \& Stapf) na zona da mata de Pernanbuco. Recife: UFRPE, 1982. 179p. Tese de Mestrado. 